Proyecciones Journal of Mathematics

Vol. 36, No 4, pp. 567-587, December 2017.

Universidad Católica del Norte

Antofagasta - Chile

\title{
On Triple sequence space of Bernstein operator of Rough $I$ - convergence pre-cauchy sequences
}

\author{
Ayhan Esi \\ Adiyaman University, Turkey \\ N. Subramanian \\ Sastra University, India \\ and \\ Ayten Esi \\ Adiyaman University, Turkey \\ Received : January 2017. Accepted : May 2017
}

\begin{abstract}
We introduce and study some basic properties of rough I- convergent pre-Cauchy sequences of triple sequence of Bernstein polynomials and also study the set of all rough I- limits of a pre-Cauchy sequence of triple sequence of Bernstein polynomials and relation between analytic ness and rough I- statistical convergence of pre-Cauchy sequence of a triple sequences of Bernstein polynomials .
\end{abstract}

Keywords : Triple sequences, rough convergence, closed and convex, cluster points and rough limit points,Bernstein polynomials, preCauchy sequences.

2010 Mathematics Subject Classification : 40F05, 40J05, 40G05. 


\section{Introduction}

The idea of rough convergence was first introduced by Phu [10-12] in finite dimensional normed spaces. He showed that the set $L I M_{x}^{r}$ is bounded, closed and convex; and he introduced the notion of rough Cauchy sequence. He also investigated the relations between rough convergence and other convergence types and the dependence of $L I M_{x}^{r}$ on the roughness of degree $r$.

Aytar [1] studied of rough statistical convergence and defined the set of rough statistical limit points of a sequence and obtained to statistical convergence criteria associated with this set and prove that this set is closed and convex. Also, Aytar [2] established that the $r$ - limit set of the sequence is equal to intersection of these sets and that $r$ - core of the sequence is equal to the union of these sets. Dündar and Cakan [9] investigated of rough ideal convergence and defined the set of rough ideal limit points of a sequence The notion of $I$ - convergence of a triple sequence which is based on the structure of the ideal $I$ of subsets of $\mathbf{N} \times \mathbf{N} \times \mathbf{N}$, where $\mathbf{N}$ is the set of all natural numbers, is a natural generalization of the notion of convergence and statistical convergence.

In this paper we study some basic properties of rough $I$ - convergence pre-Cauchy of a triple sequence of Bernstein polynomials in three dimensional cases which are not earlier. We study the set of all rough $I-$ preCauchy sequence of limits of a triple sequence of Bernstein polynomials and also the relation between analytic ness and rough $I$ - convergence of pre-Cauchy sequence of a triple sequence of Bernstein polynomials.

Let $K$ be a subset of the set of positive integers $\mathbf{N} \times \mathbf{N} \times \mathbf{N}$ and let us denote the set $K_{i k \ell}=\{(m, n, k) \in K: m \geq i, n \leq j, k \leq \ell\}$. Then the natural density of $K$ is given by

$$
\delta(K)=\lim _{i, j, \ell \rightarrow \infty} \frac{\left|K_{i j \ell}\right|}{i j \ell},
$$

where $\left|K_{i j \ell}\right|$ denotes the number of elements in $K_{i j \ell}$.

The Bernstein operator of order $(r, s, t)$ is given by

$$
\begin{gathered}
B_{r s t}(f, x) \\
=\sum_{m=0}^{r} \sum_{n=0}^{s} \sum_{k=0}^{t} f\left(\frac{m n k}{r s t}\right)(r m)(s n)(t k) x^{m+n+k}(1-x)^{(m-r)+(n-s)+(k-t)}
\end{gathered}
$$

where $f$ is a continuous (real or complex valued) function defined on $[0,1]$. 
Throughout the paper, $\mathbf{R}$ denotes the real of three dimensional space with metric $(X, d)$. Consider a triple sequence of Bernstein polynomials $\left(B_{m n k}(f, x)\right)$ such that $\left(B_{m n k}(f, x)\right) \in \mathbf{R}, m, n, k \in \mathbf{N}$.

Let $f$ be a continuous function defined on the closed interval $[0,1]$. A triple sequence of Bernstein polynomials $\left(B_{r s t}(f, x)\right)$ is said to be statistically convergent to $0 \in \mathbf{R}$, written as $s t-\lim x=0$, provided that the set

$$
K_{\epsilon}:=\left\{(m, n, k) \in \mathbf{N}^{3}:\left|B_{m n k}(f, x)-f(x)\right| \geq \epsilon\right\}
$$

has natural density zero for any $\epsilon>0$. In this case, 0 is called the statistical limit of the triple sequence of Bernstein polynomials. i.e., $\delta\left(K_{\epsilon}\right)=0$. That is,

$$
\lim _{r s t \rightarrow \infty} \frac{1}{r s t}\left|\left\{(m, n, k) \leq(r, s, t):\left|B_{m n k}(f, x)-(f, x)\right| \geq \epsilon\right\}\right|=0 .
$$

In this case, we write $\delta-\lim B_{m n k}(f, x)=f(x)$ or $B_{m n k}(f, x) \rightarrow S_{B}$ $f(x)$.

Throughout the paper, $\mathbf{N}$ denotes the set of all positive integers , $\chi_{A}-$ the characteristic function of $A \subset \mathbf{N}, \mathbf{R}$ the set of all real numbers. A subset $A$ of $\mathbf{N}$ is said to have asymptotic density $d(A)$ if

$$
d(A)=\lim _{i j \ell \rightarrow \infty} \frac{1}{i j \ell} \sum_{m=1}^{i} \sum_{n=1}^{j} \sum_{k=1}^{\ell} \chi_{A}(K) .
$$

A triple sequence (real or complex) can be defined as a function $x$ : $\mathbf{N} \times \mathbf{N} \times \mathbf{N} \rightarrow \mathbf{R}(\mathbf{C})$, where $\mathbf{N}, \mathbf{R}$ and $\mathbf{C}$ denote the set of natural numbers, real numbers and complex numbers respectively. The different types of notions of triple sequence was introduced and investigated at the initial by Sahiner et al. [13,14], Esi et al. [3-6], Datta et al. [7],Subramanian et al. [15], Debnath et al. [8], Tripathy and Goswami [16], [17], [18], [19]) and many others.

A very interesting notion was introduced, that of statistically pre-Cauchy sequences and it was shown that statistically convergent sequences are always statistically pre-Cauchy and on the other hand under certain general conditions statistical pre-Cauchy condition implies statistical convergence of a sequence (and so one do not have to guess the limit of the statistically convergent sequence before hand).

A triple sequence of Bernstein polynomials is said to be triple Bernstein polynomials pre-Cauchy of analytic if

$$
\sup _{m, n, k} \frac{1}{r^{6} s^{6} t^{6}}\left|B_{m n k}(f, x)-B_{u v w}(f, x)\right|^{\frac{1}{m+n+k}}<\infty .
$$

The space of all triple of Bernstein polynomials pre-Cauchy of analytic sequences are usually denoted by $\Lambda_{B}^{3}$. 


\section{Definitions and Preliminaries}

Throughout the paper $\mathbf{R}^{3}$ denotes the real three dimensional case with the metric. Consider a triple sequence $x=\left(x_{m n k}\right)$ such that $x_{m n k} \in$ $\mathbf{R}^{3} ; m, n, k \in \mathbf{N}$. The following definition are obtained:

\subsection{Definition}

Let $f$ be a continuous function defined on the closed interval $[0,1]$. A triple sequence of Bernstein polynomials $\left(B_{m n k}(f, x)\right)$ is said to be statistically pre-Cauchy if, for any $\epsilon>0$ and $\delta>0$,

$$
\left\{(r, s, t) \in \mathbf{N}: \frac{1}{r^{6} s^{6} t^{6}}\left|\left\{\left|B_{m n k}(f, x)-B_{u v w}(f, x)\right| \geq \epsilon\right\}\right| \geq \delta\right\} \in I .
$$

\subsection{Definition}

Let $f$ be a continuous function defined on the closed interval $[0,1]$. A triple sequence of Bernstein polynomials $\left(B_{m n k}(f, x)\right)$ is said to be statistically pre-Cauchy convergent to $B_{u v w}(f, x)$ denoted by $B_{m n k}(f, x) \rightarrow^{\text {st-limx }}$ $B_{u v w}(f, x)$, provided that the set, for any $\epsilon>0$ and $\delta>0$

$$
\left\{(r, s, t) \in \mathbf{N}: \frac{1}{r^{6} s^{6} t^{6}}\left|\left\{\left|B_{m n k}(f, x)-B_{u v w}(f, x)\right| \geq \epsilon\right\}\right| \geq \delta\right\} \in I .
$$

has natural density zero for every $\epsilon>0$.

In this case, $B_{u v w}(f, x)$ is called the statistical pre-Cauchy limit of the sequence of Berstein polynomials.

\subsection{Definition}

Let $f$ be a continuous function defined on the closed interval $[0,1]$. A triple sequence of Bernstein polynomials $\left(B_{m n k}(f, x)\right)$ in a metric space $(X,|.,|$. and $r$ be a non-negative real number is said to be pre-Cauchy $r$-convergent to $B_{\text {uvw }}(f, x)$, denoted by $B_{m n k}(f, x) \rightarrow^{r} B_{\text {uvw }}(f, x)$, if for any $\epsilon>0$ and $\delta>0$ there exists $N_{\epsilon} \in \mathbf{N}$ such that for all $m, n, k \geq N_{\epsilon}$ we have

$$
\left\{(r, s, t) \in \mathbf{N}: \frac{1}{r^{6} s^{6} t^{6}}\left|\left\{\left|B_{m n k}(f, x)-B_{u v w}(f, x)\right|<r+\epsilon\right\}\right|<\delta\right\} \in I .
$$

In this case $B_{m n k}(f, x)$ is called an pre-Cauchy $r$ - limit of $B_{u v w}(f, x)$. 


\subsection{Remark}

We consider $r$ - limit set $B_{m n k}(f, x)$ which is denoted by $L I M_{B_{m n k}(f, x)}^{r}$ and is defined by

$$
L I M_{B_{m n k}(f, x)}^{r}=\left\{B_{m n k}(f, x) \in X: B_{m n k}(f, x) \rightarrow^{r} B_{u v w}(f, x)\right\} .
$$

\subsection{Definition}

Let $f$ be a continuous function defined on the closed interval $[0,1]$. A triple sequence of Bernstein polynomials $\left(B_{m n k}(f, x)\right)$ is said to be pre-Cauchy $r$ - convergent if $\operatorname{LIM}_{B_{m n k}(f, x)}^{r} \neq \phi$ and $r$ is called a pre-Cauchy rough convergence degree of $B_{m n k}(f, x)$. If $r=0$ then it is ordinary convergence of triple sequence of Bernstein polynomials.

\subsection{Definition}

Let $f$ be a continuous function defined on the closed interval $[0,1]$. A triple sequence of Bernstein polynomials $\left(B_{m n k}(f, x)\right)$ in a metric space $(X,|.,|$.$) and r$ be a non-negative real number is said to be $r$ - statistically pre-Cauchy convergent to $B_{\text {uvw }}(f, x)$, denoted by $B_{m n k} f(x) \rightarrow^{r-s t_{3}}$ $B_{u v w}(f, x)$, if for any $\epsilon>0$ and $\delta>0$

$$
\left\{(r, s, t) \in \mathbf{N}: \frac{1}{r^{6} s^{6} t^{6}}\left|\left\{\left|B_{m n k}(f, x)-B_{u v w}(f, x)\right| \geq r+\epsilon\right\}\right|<\delta\right\} \in I .
$$

In this case $B_{u v w}(f, x)$ is called $r$ - statistical pre-Cauchy limit of $B_{m n k}(f, x)$. If $r=0$ then it is ordinary statistical convergent of triple sequence of Bernstein polynomials.

\subsection{Definition}

A class $I$ of subsets of a nonempty set $X$ is said to be an ideal in $X$ provided

(i) $\phi \in I$

(ii) $A, B \in I$ implies $A \cup B \in I$.

(iii) $A \in I, B \subset A$ implies $B \in I$.

$I$ is called a nontrivial ideal if $X \notin I$.

\subsection{Definition}

A nonempty class $F$ of subsets of a nonempty set $X$ is said to be a filter in $X$. Provided 
(i) $\phi \in F$.

(ii) $A, B \in F$ implies $A \cap B \in F$.

(iii) $A \in F, A \subset B$ implies $B \in F$.

\subsection{Definition}

$I$ is a non trivial ideal in $X, X \neq \phi$, then the class

$$
F(I)=\{M \subset X: M=X \backslash A \text { for some } A \in I\}
$$

is a filter on $X$, called the filter associated with $I$.

\subsection{Definition}

A non trivial ideal $I$ in $X$ is called admissible if $\{x\} \in I$ for each $x \in X$.

\subsection{Note}

If $I$ is an admissible ideal, then usual convergence in $X$ implies $I$ convergence in $X$.

\subsection{Remark}

If $I$ is an admissible ideal, then usual rough convergence implies rough $I-$ convergence.

\subsection{Definition}

Let $f$ be a continuous function defined on the closed interval $[0,1]$. A triple sequence of Bernstein polynomials $\left(B_{m n k}(f, x)\right)$ in a metric space $(X,|.,|$. and $r$ be a non-negative real number is said to be rough ideal convergent pre-Cauchy or $r I$ - convergent to $B_{u v w}(f, x)$, denoted by $B_{m n k} \rightarrow^{r I}$ $B_{\text {uvw }}(f, x)$, if for any $\epsilon>0$ and $\delta>0$ we have

$$
\left\{\left\{(r, s, t) \in \mathbf{N}: \frac{1}{r^{6} s^{6} t^{6}}|| B_{m n k}(f, x)-B_{u v w}(f, x) \mid \geq r+\epsilon\right\} \mid \geq \delta\right\} \in I .
$$

In this case $\left(B_{m n k}(f, x)\right)$ is called $r I$ - pre-Cauchy sequence of limit of $B_{u v w}(f, x)$ and a triple sequence of Bernstein polynomials $\left(B_{m n k}(f, x)\right)$ is called rough $I$ - convergent of pre-Cauchy sequence of $B_{u v w}(f, x)$ with $r$ as roughness pre-Cauchy sequences of degree. If $r=0$ then it is ordinary $I$ - convergent of pre-Cauchy sequences. 


\subsection{Note}

Generally, Let $f$ be a continuous function defined on the closed interval $[0,1]$. A triple sequence of Bernstein polynomials $\left(B_{m n k}(f, y)\right)$ is not $I-$ convergent pre-Cauchy sequences in usual sense and $\left|B_{m n k}(f, x)-B_{m n k}(f, y)\right| \leq$ $r$ for all $(r, s, t) \in \mathbf{N}$ or

$$
\left\{(r, s, t) \in \mathbf{N}: \frac{1}{r^{6} s^{6} t^{6}}|| B_{m n k}(f, x)-B_{m n k}(f, y)|\geq r| \geq \delta\right\} \in I .
$$

for some $r>0$. Then the triple sequence of Bernstein polynomials $\left(B_{m n k}(f, x)\right)$ is $r I$ - convergent pre-Cauchy sequences.

\subsection{Note}

It is clear that $r I$ - pre-Cauchy sequence of limit of $B_{u v w}(f, x)$ is not necessarily unique.

\subsection{Definition}

Consider $r I$ - pre-Cauchy sequence limit set of $B_{u v w}(f, x)$, which is denoted by

$$
I-L I M_{B_{m n k}(f, x)}^{r}=\left\{L \in X: B_{m n k}(f, x) \rightarrow^{r I} B_{u v w}(f, x)\right\},
$$

then the triple sequence of Bernstein polynomials $\left(B_{m n k}(f, x)\right)$ is said to be $r I$ - convergent pre-Cauchy sequences, if $I-L I M_{B_{m n k}(f, x)}^{r} \neq \phi$ and $r$ is called a rough $I$ - convergence pre-Cauchy sequences of degree of $B_{m n k}(f, x)$.

\subsection{Definition}

Let $f$ be a continuous function defined on the closed interval $[0,1]$. A triple sequence of Bernstein polynomials $\left(B_{m n k}(f, x)\right)$ is said to be $I$ - preCauchy sequences of analytic if there exists a positive real number $M$ such that

$$
\left\{(m, n, k) \in \mathbf{N}:\left|B_{m n k}(f, x)\right|^{1 / m+n+k} \geq M\right\} \in I .
$$




\subsection{Definition}

A point $L \in X$ is said to be an $I$ - pre-Cauchy sequences of accumulation point and Let $f$ be a continuous function defined on the closed interval $[0,1]$. A triple sequence of Bernstein polynomials $\left(B_{m n k}(f, x)\right)$ in a metric space $(X, d)$ if and only if for each $\epsilon>0$ and $\delta>0$, the set

$\left\{(r, s, t) \in \mathbf{N}:\left|d\left(B_{m n k}(f, x), B_{u v w}(f, x)\right)=\right| B_{m n k}(f, x)-B_{u v w}(f, x) \mid<\right.$ $\epsilon<\delta \notin I$.

We denote the set of all $I$ - pre-Cauchy sequence of accumulation points of $\left(B_{m n k}(f, x)\right)$ by $I\left(\Gamma_{B_{m n k}(f, x)}\right)$.

\subsection{Definition}

Let $f$ be a continuous function defined on the closed interval $[0,1]$. A triple sequence of Bernstein polynomials $\left(B_{m n k}(f, x)\right)$ of real numbers, the notions of ideal pre-Cauchy sequences of limit superior and ideal pre-Cauchy sequences of limit inferior are defined as follows:

$$
I-\text { limsup } B_{m n k}(f, x)=\left\{\begin{array}{cc}
\sup B_{x}, & \text { if } B_{B_{m n k}(f, x)} \neq \phi \\
-\infty, & \text { if } B_{B_{m n k}(f, x)}=\phi
\end{array}\right\},
$$

and

$I-\liminf \left(B_{m n k}(f, x)\right)=\left\{\begin{array}{cc}\inf A_{B_{m n k}(f, x),} & \text { if } A_{B_{m n k}(f, x)} \neq \phi, \\ +\infty, & \text { if } A_{B_{m n k}(f, x)}=\phi\end{array}\right\}$,

where $A_{B_{m n k}(f, x)}=\left\{a \in \mathbf{R}:\left\{(m, n, k) \in \mathbf{N}: B_{m n k}(f, x)<a\right\} \notin I\right\}$ and $B_{B_{m n k}(f, x)}=\left\{b \in \mathbf{R}:\left\{(m, n, k) \in \mathbf{N}^{3}: B_{m n k}(f, x)>b\right\} \notin I\right\}$.

\subsection{Definition}

Let $f$ be a continuous function defined on the closed interval $[0,1]$. A triple sequence of Bernstein polynomials $\left(B_{m n k}(f, x)\right)$ is said to be rough $I$ - convergent pre-Cauchy sequences if $I-L I M^{r} B_{m n k}(f, x) \neq \phi$. It is clear that if $I-L I M^{r} B_{m n k}(f, x) \neq \phi$ for a triple sequence of Bernstein polynomials $\left(B_{m n k}(f, x)\right)$ of real numbers, then we have

$\operatorname{I-LIM}^{r} B_{m n k}(f, x)=\left[I-\limsup B_{m n k}(f, x)-r, I-\liminf B_{m n k}(f, x)+r\right]$. 


\section{Main Results}

\subsection{Theorem}

Let $f$ be a continuous function defined on the closed interval $[0,1]$. A triple sequence of Bernstein polynomials $\left(B_{m n k}(f, x)\right)$ of real numbers and $I \subset 2^{\mathrm{N}}$ be an admissible ideal of pre-Cauchy sequences, we have diam $\left(I-L I M^{r} B_{m n k}(f, x)\right) \leq 2 r$. In general, diam $\left(I-L I M^{r} B_{m n k}(f, x)\right)$ has an upper bound.

Proof: Assume that diam $\left(L I M^{r} B_{m n k}(f, x)\right)$. Then, $\exists p, q \in L I M^{r} B_{m n k}(f, x) \ni$ : $|p-q|>2 r$. Take $\epsilon \in\left(0, \frac{|p-q|}{2}-r\right)$. Because $p, q \in I-L I M^{r} B_{m n k}(f, x)$, we have $A_{1}(\epsilon) \in I$ and $A_{2}(\epsilon) \in I$ for every $\epsilon>0$, where $A_{1}(\epsilon)=\left\{(r, s, t) \in \mathbf{N}: \frac{1}{r^{6} s^{6} t^{6}}|| B_{m n k}(f, x)-p|\geq r+\epsilon| \geq \delta\right\}$ and $A_{2}(\epsilon)=\left\{(r, s, t) \in \mathbf{N}: \frac{1}{r^{6} s^{6} t^{6}}|| B_{m n k}(f, x)-q|\geq r+\epsilon| \geq \delta\right\}$.

Using the properties $F(I)$, we get

$$
\left(A_{1}(\epsilon)^{c} \bigcap A_{2}(\epsilon)^{c}\right) \in F(I) .
$$

Thus we write, $|p-q| \leq\left|B_{m n k}(f, x)-p\right|+\left|B_{m n k}(f, x)-q\right|<(r+\epsilon)+$ $(r+\epsilon)<2 \delta<2(r+\epsilon)$, for all $(m, n, k) \in A_{1}(\epsilon)^{c} \cap A_{2}(\epsilon)^{c}$ which is a contradiction. Hence diam $\left(\operatorname{LIM}^{r} B_{m n k}(f, x)\right) \leq 2 r$.

Now, consider a triple sequence of Bernstein polynomials of $\left(B_{m n k}(f, x)\right)$ of real numbers such that $I-\lim _{m n k \rightarrow \infty} B_{m n k}(f, x)=B_{u v w}(f, x)$.

Let $\epsilon>0$. Then we can write

$$
\left\{(r, s, t) \in \mathbf{N}: \frac{1}{r^{6} s^{6} t^{6}}\left|\left\{\left|B_{m n k}(f, x)-B_{u v w}(f, x)\right| \geq \epsilon\right\}\right| \geq \delta\right\} \in I .
$$

Thus, we have

$$
\begin{aligned}
\mid B_{m n k}(f, x)- & p|\leq| B_{m n k}(f, x)-B_{u v w}(f, x)|+| B_{u v w}(f, x)-p \mid \\
\leq & \left|B_{m n k}(f, x)-B_{u v w}(f, x)\right|+r+\epsilon,
\end{aligned}
$$

for each $p \in \bar{B}_{r}\left(B_{u v w}(f, x)\right):=\left\{p \in \mathbf{R}^{3}:\left|p-B_{u v w}(f, x)\right| \leq r\right\}$. Then, we get

$$
\left|B_{m n k}(f, x)-p\right|<r+\epsilon
$$


for each $(r, s, t) \in\left\{(r, s, t) \in \mathbf{N}: \frac{1}{r^{6} s^{6} t^{6}}|| B_{m n k}(f, x)-B_{u v w}(f, x)|<\epsilon|<\delta\right\}$. Because the triple sequence of Bernstein polynomials of $B_{m n k}(f, x)$ is $I-$ convergent pre-Cauchy sequences to $B_{u v w}(f, x)$, we have

$$
\left\{(r, s, t) \in \mathbf{N}: \frac{1}{r^{6} s^{6} t^{6}}|| B_{m n k}(f, x)-B_{u v w}(f, x)|<\epsilon|<\delta\right\} \in F(I) .
$$

Therefore, we get $p \in I-L I M^{r} B_{m n k}(f, x)$. Consequently, we can write

$$
I-L I M^{r} B_{m n k}(f, x)=\bar{B}_{r}\left(B_{u v w}(f, x)\right) .
$$

Because diam $\left(\bar{B}_{r}\left(B_{u v w}(f, x)\right)\right)=2 r$, this shows that in general, the upper bound $2 r$ of the diameter of the set $I-L I M^{r} B_{m n k}(f, x)$ is not lower bound.

\subsection{Theorem}

Let $I \subset 3^{\mathbf{N}}$ be an admissible ideal and Let $f$ be a continuous function defined on the closed interval $[0,1]$. A triple sequence of Bernstein polynomials of $\left(B_{m n k}(f, x)\right)$ is pre-Cauchy $I-$ analytic if and only if there exists a nonnegative real number $r$ such that $I-L I M^{r} B_{m n k}(f, x) \neq \phi$, for all $r>0$, an $I$ - analytic pre-Cauchy triple sequence of Bernstein polynomials always contains a sub sequence $\left(B_{m_{i} n_{j} k_{\ell}}(f, x)\right)$ with $I-L I M^{\left(B_{m_{i} n_{j} k_{\ell}}(f, x), r\right)} \neq \phi$.

Proof: Because the triple sequence of Bernstein polynomials of $B_{m n k}(f, x)$ is Pre-Cauchy $I$ - analytic then there exists a positive real number $M$ such that

$$
\left\{(r, s, t) \in \mathbf{N}: \frac{1}{r^{6} s^{6} t^{6}}\left|\left\{\left|B_{m n k}(f, x)\right|^{1 / m+n+k} \geq M\right\}\right| \geq \delta\right\} \in I .
$$

Define $r^{\prime}=\sup \left\{\mathbf{N}^{3}:\left|B_{m n k}(f, x)\right|^{1 / m+n+k} \geq M:(m, n, k) \in K^{c}\right\}$, where $K=\left\{(m, n, k) \in \mathbf{N}^{3}:\left|B_{m n k}(f, x)\right|^{1 / m+n+k} \geq M\right\}$. Then the set $I-$ $L I M^{r^{\prime}} B_{m n k}(f, x)$ contains the origin of $\mathbf{R}^{3}$. So we have $I-L I M^{r^{\prime}} B_{m n k}(f, x) \neq$ $\phi$.

If $I-L I M^{r} B_{m n k}(f, x) \neq \phi$ for some $r \geq 0$, then there exists $B_{u v w}(f, x)$ such that $L \in I-L I M^{r} B_{m n k}(f, x)$, i.e.,

$$
\left\{(r, s, t) \in \mathbf{N}: \frac{1}{r^{6} s^{6} t^{6}}\left|\left\{\left|B_{m n k}(f, x)-B_{u v w}(f, x)\right|^{1 / m+n+k} \geq r+\epsilon\right\}\right| \geq \delta\right\} \epsilon
$$


for each $\epsilon>0$. Then we say that almost all $B_{m n k}(f, x)$ are contained in some ball with any radius greater than $r$. So the triple sequence space of Bernstein polynomials of $B_{m n k}(f, x)$ is $I$ - analytic.

As $B_{m n k}(f, x)$ is a $I$ - analytic triple sequence of Bernstein polynomials in a three-dimensional metric space, it certainly contains a $I$ - convergent sub sequence $\left(B_{m_{i} n_{j} k_{\ell}}(f, x)\right)$. Let $f(x)$ be its $I$ - limit point, then $I-$ $L I M^{r} B_{m_{i} n_{j} k_{\ell}}(f, x)=\bar{B}_{r}(f(x))$ and, for $r>0$,

$$
I-L I M^{\left(B_{m_{i} n_{j} k_{\ell}}(f, x)\right), r} B_{m_{i} n_{j} k_{\ell}}(f, x) \neq \phi .
$$

\subsection{Theorem}

Let $I \subset 3^{\mathbf{N}}$ be an pre-Cauchy admissible ideal. If $\left(B_{m_{i} n_{j} k_{\ell}}(f, x)\right)$ is a pre-Cauchy sub sequence of $\left(B_{m n k}(f, x)\right)$, then

$$
I-L I M^{r} B_{m n k}(f, x) \subseteq I-L I M^{r} B_{m_{i} n_{j} k_{\ell}}(f, x) .
$$

Proof: The proof is trivial (See [10], Proposition 2.3).

\subsection{Theorem}

Let $f$ be a continuous function defined on the closed interval $[0,1]$. A triple sequence of Bernstein polynomials of $\left(B_{m n k}(f, x)\right)$ and $I \subset 3^{\mathbf{N}}$ be an preCauchy admissible ideal is closed.

Proof: The result is true for $I-L I M^{r} B_{m n k}(f, x)=\phi$. Assume that $I-L I M^{r} B_{m n k}(f, x) \neq \phi$.

Then, we can choose a triple sequence of Bernstein polynomials of $B_{m n k}(f, y) \subseteq I-L I M^{r} B_{m n k}(f, x)$ such that $B_{m n k}(f, y) \rightarrow^{r} B_{u v w}(f, y)$ for $m, n, k \rightarrow \infty$. To prove $f(x) \in I-L I M^{r} B_{m n k}(f, x)$.

Let $\epsilon>0$ be given. Because $B_{m n k}(f, y) \rightarrow B_{u v w}(f, y), \exists i, j, \ell=$ $i_{\frac{\epsilon}{2}}, j_{\frac{\epsilon}{2}}, \ell_{\frac{\epsilon}{2}} \in \mathbf{N}^{3}$ such that

$$
\left|B_{m n k}(f, y)-B_{u v w}(f, y)\right|<\frac{\epsilon}{2}, \forall m \geq i_{\frac{\epsilon}{2}}, n \geq j_{\frac{\epsilon}{2}}, k \geq \ell_{\frac{\epsilon}{2}} .
$$

Now choose an $m_{0} n_{0} k_{0} \in \mathbf{N}^{3}$ such that $m_{0} \geq i_{\frac{\epsilon}{2}}, n_{0} \geq j_{\frac{\epsilon}{2}}, k_{0} \geq \ell_{\frac{\epsilon}{2}}$.

Then we can write

$$
\left|B_{m_{0} n_{0} k_{0}}(f, y)-B_{u_{0} v_{0} w_{0}}(f, y)\right|<\frac{\epsilon}{2} .
$$


On the other hand, because $B_{m n k}(f, y) \subseteq I-L I M^{r} B_{m n k}(f, x)$, we have $B_{m_{0} n_{0} k_{0}}(f, y) \in I-L I M^{r} B_{m n k}(f, x)$, namely,

$\left\{(r, s, t) \in \mathbf{N}: \frac{1}{r^{6} s^{6} t^{6}}\left|\left\{\left|B_{m n k}(f, x)-B_{m_{0} n_{0} k_{0}}(f, y)\right| \geq r+\frac{\epsilon}{2}\right\}\right| \geq \delta\right\} \in I$.

Now let us prove that the inclusion

$$
A^{c}\left(\frac{\epsilon}{2}\right) \subseteq A^{c}(\epsilon)
$$

holds, where

$$
A(\epsilon)=\left\{(r, s, t) \in \mathbf{N}: \frac{1}{r^{6} s^{6} t^{6}}\left|\left\{\left|B_{m n k}(f, x)-B_{u v w}(f, x)\right| \geq r+\epsilon\right\}\right| \geq \delta\right\} \in
$$

Take $(u, v, w) \in A^{c}\left(\frac{\epsilon}{2}\right)$. Then we have

$$
\left|B_{u v w}(f, x)-B_{m_{0} n_{0} k_{0}}(f, y)\right|<r+\frac{\epsilon}{2}
$$

and hence

$$
\begin{gathered}
\left|B_{u v w}(f, x)-B_{u v w}(f, x)\right| \leq \\
\left|B_{u v w}(f, x)-B_{m_{0} n_{0} k_{0}}(f, y)\right|+\left|B_{m_{0} n_{0} k_{0}}(f, y)-B_{u v w}(f, x)\right|<r+\epsilon,
\end{gathered}
$$

i.e., $(u, v, w) \in A^{c}(\epsilon)$, which proves (3.3), we get $A(\epsilon) \in I$

(i.e., $\left.B_{\text {uvw }}(f, x) \in I-L I M^{r} B_{m n k}(f, x)\right)$.

\subsection{Theorem}

Let $f$ be a continuous function defined on the closed interval $[0,1]$. A triple sequence of Bernstein polynomials of $\left(B_{m n k}(f, x)\right)$ of real numbers and $I \subset 2^{\mathbf{N}}$ be an pre-Cauchy admissible ideal. The pre-Cauchy rough $I$ - limit set of triple sequence of Bernstein polynomials of $B_{m n k}(f, x)$ is convex.

Proof: Let $y_{1}, y_{2} \in B_{m n k}(f, x) \in I-L I M^{r} B_{m n k}(f, x)$ for triple sequence of Bernstein polynomials of $B_{m n k}(f, x)$ and let $\epsilon>0$ be given. Define

$$
A_{1}(\epsilon)=\left\{(r, s, t) \in \mathbf{N}: \frac{1}{r^{6} s^{6} t^{6}}\left|\left\{\left|B_{m n k}(f, x)-y_{1}\right| \geq r+\epsilon\right\}\right| \geq \delta\right\} \in I .
$$

and 


$$
A_{2}(\epsilon)=\left\{(r, s, t) \in \mathbf{N}: \frac{1}{r^{6} s^{6} t^{6}}\left|\left\{\left|B_{m n k}(f, x)-y_{2}\right| \geq r+\epsilon\right\}\right| \geq \delta\right\} \in I .
$$

Because $y_{1}, y_{2} \in I-L I M^{r} B_{m n k}(f, x)$, we have $A_{1}(\epsilon), A_{2}(\epsilon) \in I$. Thus we have

$$
\begin{aligned}
\left|B_{m n k}(f, x)-\left[(1-\lambda) y_{1}+\lambda y_{2}\right]\right| & =\left|(1-\lambda)\left(B_{m n k}(f, x)-y_{1}\right)+\lambda\left(B_{m n k}(f, x)-y_{2}\right)\right| \\
& \leq(1-\lambda)\left|B_{m n k}(f, x)-y_{1}\right|+\lambda\left|B_{m n k}(f, x)-y_{2}\right| \\
& <(1-\lambda)(r+\epsilon)+\lambda(r+\epsilon)<r+\epsilon
\end{aligned}
$$

for each $(m, n, k) \in A_{1}^{c}(\epsilon) \bigcap A_{2}^{c}(\epsilon)$ and each $\lambda \in[0,1]$. Because $\left(A_{1}^{c}(\epsilon) \bigcap A_{2}^{c}(\epsilon)\right) \in$ $F(I)$ by definition of $F(I)$, we get

$$
\left\{(r, s, t) \in \mathbf{N}: \frac{1}{r^{6} s^{6} t^{6}}\left|\left\{\left|B_{m n k}(f, x)-\left[(1-\lambda) y_{1}+\lambda y_{2}\right]\right| \geq r+\epsilon\right\}\right| \geq \delta\right\} \in
$$

that is

$$
\left[(1-\lambda) y_{1}+\lambda y_{2}\right] \in I-L I M^{r} B_{m n k}(f, x),
$$

which proves the convexity of the set $I-L I M^{r} B_{m n k}(f, x)$.

\subsection{Theorem}

Let $f$ be a continuous function defined on the closed interval $[0,1]$. A triple sequence of Bernstein polynomials of $\left(B_{m n k}(f, x)\right)$ of real numbers $r>0$ and $I \subset 3^{\mathbf{N}}$ be an pre-Cauchy admissible ideal is rough $I-$ convergent to $B_{u v w}(f, x)$ if and only if there exists a triple sequence of Bernstein polynomials of $B_{m n k}(f, y)$ such that

$$
I-\lim B_{m n k}(f, y)=B_{u v w}(f, y) \text { and }\left|B_{m n k}(f, x)-B_{m n k}(f, y)\right| \leq r,
$$

$$
\text { for each } m, n, k \in \mathbf{N}^{3} \text {. }
$$

Proof: Assume that the triple sequence of Bernstein polynomials of $B_{m n k}(f, x)$ is rough $I$ - convergent to $B_{u v w}(f, x)$. Then we have

$$
I-\limsup \left|B_{m n k}(f, x)-B_{u v w}(f, x)\right| \leq r .
$$

Now, define

$$
\begin{gathered}
B_{m n k}(f, y)=\left\{B_{u v w}(f, x), \text { if }\left|B_{m n k}(f, x)-B_{u v w}(f, x)\right| \leq r,\right. \\
\mathrm{B}_{m n k}(f, x)+r\left(\frac{B_{u v w}(f, x)-B_{m n k}(f, x)}{\left|B_{m n k}(f, x)-B_{u v w}(f, x)\right|}\right), \text { otherwise }
\end{gathered}
$$

Then, we we write 


$$
\begin{gathered}
\left|B_{m n k}(f, x)-B_{u v w}(f, x)\right|= \\
\left\{\mid B_{u v w}(f, x)-B_{u v w}(f, x), \text { if }\left|B_{m n k}(f, x)-B_{u v w}(f, x)\right| \leq r,\right. \\
\left|B_{m n k}(f, x)-B_{u v w}(f, x)\right|+ \\
r\left(\frac{\left|B_{u v w}(f, x)-B_{u v w}(f, x)\right|-\left|B_{m n k}(f, x)-B_{u v w}(f, x)\right|}{\left|B_{m n k}(f, x)-B_{u v w}(f, x)\right|}\right), \text { otherwise }
\end{gathered}
$$

(i.e) $\left|B_{m n k}(f, y)-B_{u v w}(f, y)\right|=\left\{0\right.$, if $\left|B_{m n k}(f, x)-B_{u v w}(f, x)\right| \leq r$, $\left|B_{m n k}(f, x)-B_{u v w}(f, x)\right|-r\left(\frac{\left|B_{m n k}(f, x)-B_{u v w}(f, x)\right|}{\left|B_{m n k}(f, x)-B_{u v w}(f, x)\right|}\right)$, otherwise,

(i.e) $\left|B_{m n k}(f, y)-f(y)\right|=\left\{0\right.$, if $\left|B_{m n k}(f, x)-B_{u v w}(f, x)\right| \leq r$, $\left|B_{m n k}(f, x)-B_{u v w}(f, x)\right|-r$, otherwise.

We have $\left|B_{m n k}(f, y)-B_{\text {uvw }}(f, y)\right| \geq\left|B_{m n k}(f, x)-B_{u v w}(f, x)\right|-r \Longrightarrow$ $\left|B_{m n k}(f, x)-B_{u v w}(f, x)-B_{m n k}(f, y)+B_{u v w}(f, y)\right| \leq r$

$$
\left|B_{m n k}(f, x)-B_{m n k}(f, y)\right| \leq r
$$

for all $m, n, k \in \mathbf{N}^{3}$. By equation (3.5) and by definition of $B_{m n k}(f, y)$, we get $I-$ limsup $\left|B_{m n k}(f, x)-B_{u v w}(f, y)\right|=0$.

$\Longrightarrow I-B_{m n k}(f, y) \rightarrow^{r} B_{u v w}(f, y)$.

Assume that (3.4) holds. Because $I-\lim B_{m n k}(f, y)=B_{u v w}(f, y)$, we have

$$
A(\epsilon)=\left\{(m, n, k) \in \mathbf{N}^{3}:\left|B_{m n k}(f, y)-B_{u v w}(f, y)\right| \geq r+\epsilon\right\} \in I,
$$

for each $\epsilon>0$. Now, define the set

$$
B(\epsilon)=\left\{(m, n, k) \in \mathbf{N}^{3}:\left|B_{m n k}(f, x)-B_{u v w}(f, x)\right| \geq r+\epsilon\right\} \in I .
$$

We have

$$
B(\epsilon) \subseteq A(\epsilon)
$$

holds. Since $A(\epsilon) \in I \Longrightarrow B(\epsilon) \in I$. Hence, $B_{m n k}(f, x)$ is rough $I-$ convergent to $B_{\text {uvw }}(f, x)$.

\subsection{Note}

If we replace the condition $\left|B_{m n k}(f, x)-B_{m n k}(f, y)\right| \leq r$ for all $(m, n, k) \in$ $\mathbf{N}^{3}$ in the hypothesis of the above theorem with the condition

$\left\{(m, n, k) \in \mathbf{N}^{3}:\left|B_{m n k}(f, x)-B_{m n k}(f, y)\right|>r\right\} \in I$ then the theorem will also be valid. 


\subsection{Theorem}

Let $f$ be a continuous function defined on the closed interval $[0,1]$. A triple sequence of Bernstein polynomials of $\left(B_{m n k}(f, x)\right)$ of real numbers and $I \subset 3^{\mathbf{N}}$ be an pre-Cauchy admissible ideal. For an arbitrary $c \in I\left(\Gamma_{x}\right)$, we have $|f(x)-c| \leq r$ for all $f(x) \in I-L I M^{r} B_{m n k}(f, x)$.

Proof: Assume on the contrary that there exist a point $c \in I\left(\Gamma_{x}\right)$ and $f(x) \in I-L I M^{r} B_{m n k}(f, x)$ such that $\left|B_{\text {uvw }}(f, x)-c\right|>r$. Define $\epsilon:=$ $\frac{\left|B_{u v w}(f, x)-c\right|-r}{3}$. Then

$$
\begin{aligned}
& \left\{(r, s, t) \in \mathbf{N}: \frac{1}{r^{6} s^{6} t^{6}}\left|\left\{\left|B_{m n k}(f, x)-c\right|<\epsilon\right\}\right|<\delta\right\} \subseteq \\
& \left\{(r, s, t) \in \mathbf{N}: \frac{1}{r^{6} s^{6} t^{6}}\left|\left\{\left|B_{m n k}(f, x)-B_{u v w}(f, x)\right| \geq r+\epsilon\right\}\right| \geq \delta\right\} \in I \ldots
\end{aligned}
$$

Since $c \in I\left(\Gamma_{x}\right)$, we have

$$
\left\{(r, s, t) \in \mathbf{N}: \frac{1}{r^{6} s^{6} t^{6}}\left|\left\{\left|B_{m n k}(f, x)-c\right|<\epsilon\right\}\right|<\delta\right\} \notin I .
$$

But from definition of $I-$ convergence, since

$$
\left\{(r, s, t) \in \mathbf{N}: \frac{1}{r^{6} s^{6} t^{6}}\left|\left\{\left|B_{m n k}(f, x)-B_{u v w}(f, x)\right| \geq r+\epsilon\right\}\right| \geq \delta\right\} \in I .
$$

so by (1) we have

$$
\left\{(r, s, t) \in \mathbf{N}: \frac{1}{r^{6} s^{6} t^{6}}\left|\left\{\left|B_{m n k}(f, x)-c\right|<\epsilon\right\}\right|<\delta\right\} \in I .
$$

which contradicts the fact $c \in I\left(\Gamma_{x}\right)$. On the other hand, if $c \in I\left(\Gamma_{x}\right)$ i.e.,

$$
\left\{(r, s, t) \in \mathbf{N}: \frac{1}{r^{6} s^{6} t^{6}}\left|\left\{\left|B_{m n k}(f, x)-c\right|<\epsilon\right\}\right|<\delta\right\} \notin I .
$$

then

$$
\left\{(r, s, t) \in \mathbf{N}: \frac{1}{r^{6} s^{6} t^{6}}\left|\left\{\left|B_{m n k}(f, x)-B_{u v w}(f, x)\right| \geq r+\epsilon\right\}\right| \geq \delta\right\} \notin I,
$$

which contradicts the fact $B_{u v w}(f, x) \in I-L I M^{r} B_{m n k}(f, x)$. 


\subsection{Theorem}

Let $f$ be a continuous function defined on the closed interval $[0,1]$. A triple sequence of Bernstein polynomials of $\left(B_{m n k}(f, x)\right)$ of real numbers and $I \subset 3^{\mathbf{N}}$ be an pre-Cauchy admissible ideal, $\left(\mathbf{R}^{3},|.,|.\right)$ be a strictly convex, if there exist $y_{1}, y_{2}, y_{3}, y_{4}, y_{5}, y_{6} \in I-L I M^{r} B_{m n k}(f, x)$ such that $\left|y_{1}-y_{2}\right|<2 r,\left|y_{3}-y_{4}\right|<2 r$ and $\left|y_{5}-y_{6}\right|<2 r$, then this triple sequence space of Bernstein polynomials is $I-$ convergent to

$$
\frac{1}{6}\left(y_{1}+y_{2}+y_{3}+y_{4}+y_{5}+y_{6}\right) \text {. }
$$

Proof: Let $c \in I\left(\Gamma_{x}\right)$. Then since $y_{1}, y_{2}, y_{3}, y_{4}, y_{5}, y_{6} \in I-L I M^{r} B_{m n k}(f, x)$, we

have

$\left|y_{1}-c\right| \leq r,\left|y_{2}-c\right| \leq r,\left|y_{3}-c\right| \leq r,\left|y_{4}-c\right| \leq r,\left|y_{5}-c\right| \leq r$ and $\left|y_{6}-c\right| \leq r$

By Theorem (3.9). On the other hand, we have

$$
6 r=\left|y_{1}-y_{6}\right| \leq\left|y_{1}-c\right|+\left|y_{2}-c\right|+\left|y_{3}-c\right|+\left|y_{4}-c\right|+\left|y_{5}-c\right|+\left|y_{6}-c\right|
$$

Therefore, we get $\left|y_{1}-c\right|=\cdots=\left|y_{6}-c\right|=r$ by inequalities (3.7) and (3.8). Since

$$
\frac{1}{6}\left(y_{6}-y_{1}\right)=\frac{1}{6}\left[\left(c-y_{1}\right)+\left(c-y_{2}\right)\left(c-y_{3}\right)\left(c-y_{4}\right)+\left(c-y_{5}\right)+\left(c-y_{6}\right)\right]
$$

$$
\text { and }\left|y_{1}-y_{6}\right|=6 r \text {, }
$$

we get $\left|\frac{1}{6}\left(y_{6}-y_{1}\right)\right|=r$. By the strict convexity of the space from the equality (3.9), we get

$$
\frac{1}{6}\left(y_{6}-y_{1}\right)=c-y_{1}=\cdots=c-y_{6},
$$

which implies that $c=\frac{1}{6}\left(y_{1}+y_{2}+y_{3}+y_{4}+y_{5}+y_{6}\right)$. Hence $c$ is the unique $I$ - cluster point of the triple sequence space of Bernstein polynomials of $\left(B_{m n k}(f, x)\right)$.

On the other hand, the assumption $y_{1}, y_{2}, y_{3}, y_{4}, y_{5}, y_{6} \in I-L I M^{r} B_{m n k}(f, x) \Longrightarrow I-L I M^{r} B_{m n k}(f, x) \neq \phi$. 
By theorem (3.3), the triple sequence space of Bernstein polynomials of $B_{m n k}(f, x)$ is $I$ - analytic. Consequently, the triple sequence space of Bernstein polynomials of $B_{m n k}(f, x)$ must $I$ - convergent to

$\frac{1}{6}\left(y_{1}+y_{2}+y_{3}+y_{4}+y_{5}+y_{6}\right)$, i.e., $I-\lim B_{m n k}(f, x)=\frac{1}{6}\left(y_{1}+y_{2}+y_{3}+y_{4}+y_{5}+y_{6}\right)$.

\subsection{Theorem}

Let $f$ be a continuous function defined on the closed interval $[0,1]$. A triple sequence of Bernstein polynomials of $\left(B_{m n k}(f, x)\right)$ of real numbers and $I \subset 3^{\mathbf{N}}$ be an pre-Cauchy admissible ideal.

(i) If $c \in I\left(\Gamma_{x}\right)$ then

$$
I-L I M^{r} B_{m n k}(f, x) \subseteq \bar{B}_{r}(c) .
$$

$I-L I M^{r} B_{m n k}(f, x)=\bigcap_{c \in I\left(\Gamma_{x}\right)} \bar{B}_{r}(c)=\left\{B_{u v w}(f, x) \in \mathbf{R}^{3}: I\left(\Gamma_{x}\right) \subseteq \bar{B}_{r}(L)\right\}$.

\section{Proof:}

(i) If $c \in I\left(\Gamma_{x}\right)$ then by Theorem (3.9), we have $\left|B_{\text {uvw }}(f, x)-c\right| \leq r$ for all $B_{\text {uvw }}(f, x) \in I-L I M^{r} B_{m n k}(f, x)$, other wise we get

$$
\left\{(r, s, t) \in \mathbf{N}: \frac{1}{r^{6} s^{6} t^{6}}\left|\left\{\left|B_{m n k}(f, x)-B_{u v w}(f, x)\right| \geq r+\epsilon\right\}\right| \geq \delta\right\} \notin I .
$$

for $\epsilon:=\frac{\left|B_{u v w}(f, x)-c\right|-r}{3}$. Because $c$ is an $I-$ cluster point of $B_{m n k}(f, x)$, this contradicts with the fact that $B_{u v w}(f, x) \in I-L I M^{r} B_{m n k}(f, x)$.

(ii) From (3.11) we have

$$
I-L I M^{r} B_{m n k}(f, x) \subseteq \bigcap_{c \in I\left(\Gamma_{x}\right)} \bar{B}_{r}(c) .
$$

Now, let $B_{m n k}(f, x) \in \bigcap_{c \in I\left(\Gamma_{x}\right)} \bar{B}_{r}(c)$. Then we have

$$
\left\{(r, s, t) \in \mathbf{N}: \frac{1}{r^{6} s^{6} t^{6}}\left|\left\{\left|B_{m n k}(f, x)-c\right| \leq r\right\}\right| \leq \delta\right\} \in I .
$$


for all $c \in I\left(\Gamma_{x}\right)$, which is equivalent to $I\left(\Gamma_{x}\right) \subseteq \bar{B}_{r}\left(B_{m n k}(f, y)\right)$, i.e.,

$$
\bigcap_{c \in I\left(\Gamma_{x}\right)} \bar{B}_{r}(c)=\left\{f(x) \in \mathbf{R}^{3}: I\left(\Gamma_{x}\right) \subseteq \bar{B}_{r}(f(x))\right\} .
$$

Now, let $B_{m n k}(f, y) \notin I-L I M^{r} B_{m n k}(f, x)$. Then, there exists an $\epsilon>0$ such that

$$
\left\{(r, s, t) \in \mathbf{N}: \frac{1}{r^{6} s^{6} t^{6}}\left|\left\{\left|B_{m n k}(f, x)-B_{u v w}(f, y)\right| \geq r+\epsilon\right\}\right| \geq \delta\right\} \notin I,
$$

$\Longrightarrow$ the existence of an $I-$ cluster point $c$ of the sequence $B_{m n k}(f, x)$ with

$$
\left\{(r, s, t) \in \mathbf{N}: \frac{1}{r^{6} s^{6} t^{6}}\left|\left\{\left|B_{m n k}(f, x)-c\right| \geq r+\epsilon\right\}\right| \geq \delta\right\} \in I,
$$

i.e.,

$$
\begin{gathered}
I\left(\Gamma_{x}\right) \bar{B}_{r}\left(B_{m n k}(f, y)\right) \text { and } B_{m n k}(f, y) \notin\left\{f(x) \in \mathbf{R}^{3}: I\left(\Gamma_{x}\right) \subseteq \bar{B}_{r}(L)\right\} . \\
\text { Hence } B_{m n k}(f, y) \in I-L I M^{r} B_{m n k}(f, x) \text { follows from } \\
B_{m n k}(f, y) \in\left\{B_{u v w}(f, x) \in \mathbf{R}^{3}: I\left(\Gamma_{x}\right) \subseteq \bar{B}_{r}\left(B_{u v w}(f, x)\right)\right\}, \text { i.e. }, \\
\left\{B_{u v w}(f, x) \in \mathbf{R}^{3}: I\left(\Gamma_{x}\right) \subseteq \bar{B}_{r}\left(B_{u v w}(f, x)\right)\right\} \subseteq I-L I M^{r} B_{m n k}(f, x) .
\end{gathered}
$$

Therefore, the inclusions (3.12)-(3.14) ensure that (3.11) holds i.e.,

$$
\begin{gathered}
I-L I M^{r} B_{m n k}(f, x) \bigcap_{c \in I\left(\Gamma_{x}\right)} \bar{B}_{r}(c)= \\
\left\{B_{u v w}(f, x) \in \mathbf{R}^{3}: I\left(\Gamma_{x}\right) \subseteq \bar{B}_{r}\left(B_{u v w}(f, x)\right)\right\} .
\end{gathered}
$$

\subsection{Theorem}

Let $I \subset 3^{\mathbf{N}}$ be an pre-Cauchy admissible ideal and Let $f$ be a continuous function defined on the closed interval $[0,1]$. A triple $I-$ analytic sequence of Bernstein polynomials of $\left(B_{m n k}(f, x)\right)$ of real numbers $r \geq \operatorname{diam}\left(I\left(\Gamma_{x}\right)\right)$, then we have have $I\left(\Gamma_{x}\right) \subseteq I-L I M^{r} B_{m n k}(f, x)$. 
Proof: Let $c \notin I-L I M^{r} B_{m n k}(f, x)$. Then there exists an $\epsilon>0$ such that

$$
\left\{(r, s, t) \in \mathbf{N}: \frac{1}{r^{6} s^{6} t^{6}}\left|\left\{\left|B_{m n k}(f, x)-c\right|^{1 / m+n+k} \geq r+\epsilon\right\}\right| \geq \delta\right\} \notin I .
$$

Since $B_{m n k}(f, x)$ is $I-$ analytic and from the inequality (3.15), there exists an $I$-cluster point $c_{1}$ such that

$$
\left|c-c_{1}\right|>r+\epsilon_{1}, \text { where } \epsilon_{1}:=\frac{\epsilon}{2} .
$$

So we get

$$
\operatorname{diam}\left(I\left(\Gamma_{x}\right)\right)>r+\epsilon_{1}
$$

The converse of this theorem is also true, i.e., if $I\left(\Gamma_{x}\right) \subseteq I-L I M^{r} B_{m n k}(f, x)$, then we have $r \geq \operatorname{diam}\left(I\left(\Gamma_{x}\right)\right)$.

Competing Interests: The authors declare that there is not any conflict of interests regarding the publication of this manuscript.

\section{References}

[1] S. Aytar, Rough statistical convergence, Numer. Funct. Anal. Optimiz, 29(3-4), pp. 291-303, (2008).

[2] S. Aytar, The rough limit set and the core of a real sequence, Numer. Funct. Anal. Optimiz, 29 (3-4), pp. 283-290, (2008).

[3] A. Esi , On some triple almost lacunary sequence spaces defined by Orlicz functions, Research and Reviews: Discrete Mathematical Structures, 1 (2), pp. 16-25, (2014).

[4] A. Esi and M. Necdet Catalbas,Almost convergence of triple sequences, Global Journal of Mathematical Analysis, 2 (1), pp. 6-10, (2014). 
[5] A. Esi and E. Savas, On lacunary statistically convergent triple sequences in probabilistic normed space,Appl.Math.and Inf.Sci., 9 (5), pp. 2529-2534, (2015).

[6] A. Esi, S. Araci and M. Acikgoz, Statistical Convergence of Bernstein Operators, Appl.Math.and Inf.Sci., 10 (6), pp. 2083-2086, (2016).

[7] A. J. Dutta A. Esi and B.C. Tripathy,Statistically convergent triple sequence spaces defined by Orlicz function ,Journal of Mathematical Analysis, 4 (2), pp. 16-22, (2013).

[8] S. Debnath, B. Sarma and B.C. Das ,Some generalized triple sequence spaces of real numbers, Journal of nonlinear analysis and optimization, Vol. 6, No. 1, pp. 71-79, (2015).

[9] E. Dündar, C. Cakan, Rough I- convergence, Demonstratio Mathematica, Accepted.

[10] H.X. Phu, Rough convergence in normed linear spaces, Numer. Funct. Anal. Optimiz, 22, pp. 199-222, (2001).

[11] H.X. Phu, Rough continuity of linear operators, Numer. Funct. Anal. Optimiz, 23, pp. 139-146, (2002).

[12] H.X. Phu , Rough convergence in infinite dimensional normed spaces, Numer. Funct. Anal. Optimiz, 24, pp. 285-301, (2003).

[13] A. Sahiner, M. Gurdal and F.K. Duden, Triple sequences and their statistical convergence, Selcuk J. Appl. Math., 8 No. (2), pp. 49-55, (2007).

[14] A. Sahiner, B.C. Tripathy , Some $I$ related properties of triple sequences, Selcuk J. Appl. Math., 9 No. (2), pp. 9-18, (2008).

[15] N. Subramanian and A. Esi, The generalized tripled difference of $\chi^{3}$ sequence spaces, Global Journal of Mathematical Analysis, 3 (2), pp. 54-60, (2015).

[16] B. C. Tripathy and R. Goswami, Vector valued multiple sequences defined by Orlicz functions, Boletim da Sociedade Paranaense de Matematica, 33 (1), pp. 76-79, (2015).

[17] B. C. Tripathy and R.Goswami, Multiple sequences in probabilistic normed spaces, Afrika Matematika, 26 (5-6), pp. 753-760, (2015). 
[18] B. C. Tripathy and R.Goswami, Fuzzy real valued p-absolutely summmable multiple sequences in probabilistic normed spaces, Afrika Matematika, 26 (7-8), pp. 1281-1289, (2015).

[19] B. C. Tripathy and R.Goswami, On triplhe sequences of real numbers in probabilistic normed spaces, Proyecciones Jour.Math., 33 (2), pp. 157-174, (2014).

\title{
Ayhan Esi \\ Department of Mathematics, Adiyaman University, 02040, Adiyaman, Turkey e-mail : aesi23@hotmail.com
}

\section{N. Subramanian}

Department of Mathematics, SASTRA University, Thanjavur-613 401, India

e-mail : nsmaths@yahoo.com

and

\author{
Ayten Esi \\ Department of Mathematics, \\ Adiyaman University, 02040, \\ Adiyaman, \\ Turkey \\ e-mail : aytenesi@yahoo.com
}

